TRANSACTIONS OF THE

AMERICAN MATHEMATICAL SOCIETY

Volume 189, 1974

\title{
PAIRS OF COMPACTA AND TRIVIAL SHAPE
}

\author{
BY \\ SIBE MARDEŠIĆ( $(1)$
}

\begin{abstract}
Let $(X, Y, A),\left(X^{\prime}, Y^{\prime}, A^{\prime}\right)$ be triples of compact Hausdorff spaces. Using ANR-systems the following is proved: $\operatorname{sh} Y=\operatorname{sh} Y^{\prime}=0, \operatorname{sh}(X, Y)=\operatorname{sh}\left(X^{\prime}, Y^{\prime}\right)$ and $\operatorname{sh} A=\operatorname{sh} A^{\prime}$ imply $\operatorname{sh}(X, Y, A)=\operatorname{sh}\left(X^{\prime}, Y^{\prime}, A^{\prime}\right)$. All results concerning the shape of decomposition spaces and addition properties of FAR's, due to $K$. Borsuk and T.A. Chapman, follow readily from this theorem. In particular, sh $X=\operatorname{sh} X^{\prime}=0$ and $\operatorname{sh} A$ $=\operatorname{sh} A^{\prime}$ imply $\operatorname{sh}(X, A)=\operatorname{sh}\left(X^{\prime}, A^{\prime}\right)$, which in view of an example of Borsuk shows that for compact metric pairs the ANR-system approach to shapes differs from the Borsuk approach.
\end{abstract}

1. Introduction. The following is a typical result proved in this paper: Let ( $Y$, $A),\left(Y^{\prime}, A^{\prime}\right)$ be pairs of compact Hausdorff spaces, let sh $Y=\operatorname{sh} Y^{\prime}=0$ and let $\operatorname{sh} A=\operatorname{sh} A^{\prime}$. Then $\operatorname{sh}(Y, A)=\operatorname{sh}\left(Y^{\prime}, A^{\prime}\right)$ and thus $\operatorname{sh}(Y / A)=\operatorname{sh}\left(Y^{\prime} / A^{\prime}\right)$. The theorem was proved in [5] in the special case when $Y=Y^{\prime}$ is the $n$-cube $I^{n}$. It was generalized by $\mathrm{K}$. Borsuk [2] to the case when both $Y$ and $Y^{\prime}$ are AR's. The assertion $\operatorname{sh}(Y / A)=\operatorname{sh}\left(Y^{\prime} / A^{\prime}\right)$ for metric compacta $Y, Y^{\prime}$ of trivial shape was proved by T. A. Chapman [4] using methods of infinite-dimensional manifolds. The more general assertion $\operatorname{sh}(Y, A)=\operatorname{sh}\left(Y^{\prime}, A^{\prime}\right)$, is here obtained for the first time.

Remark 1. Borsuk exhibited in [2] two compact metric pairs $(Y, A),\left(Y^{\prime}, A^{\prime}\right)$ with $Y \doteq Y^{\prime}$ contractible and $A$ homeomorphic to $A^{\prime}$, but such that $\operatorname{sh}(Y, A)$ $\neq \operatorname{sh}\left(Y^{\prime}, A^{\prime}\right)$. This does not contradict our assertion because our assertion refers to shapes in the sense of [9], [6] while Borsuk's assertion refers to his notion of shape. It shows, however, that the two notions of shape differ in the relative case as recently established by the author [8].

In this paper we actually prove a general result concerning triples of compacta $(X, Y, A)$ with sh $Y=0$ (Theorem 3). The above stated theorem for pairs, as well as all results on quotient spaces and FAR's proved by Chapman in [3], [4] using his method of infinite-dimensional manifolds, are easily derived from this general theorem. The theorem itself is proved by elementary means, i.e. without use of techniques of either infinite-dimensional manifolds or PL-topology.

Received by the editors November 28, 1972.

AMS (MOS) subject classifications (1970). Primary 55D99; Secondary 54B15, 54B17, 54B25, $54 \mathrm{C55}$.

Key words and phrases. Shape of pairs and triples of compacta, shape of quotient spaces, ANRsystems.

(I) This paper was written while the author was visiting the University of Pittsburgh on leave from the University of Zagreb.

Copyright $\odot 1974$, American Mathematical Society 
Throughout the paper we consider ANR-systems and their maps as defined in [9]. Beside the case of spaces and their pairs, we also use ANR-systems of triples $(\mathbf{X}, \mathbf{Y}, \mathbf{A})=\left\{(X, Y, A)_{\lambda}, p_{\lambda \lambda^{\prime}}, \Lambda\right\}$, where $(X, Y, A)_{\lambda}$ denotes the triple which consists of compact ANR's for metric spaces $X_{\lambda} \supset Y_{\lambda} \supset A_{\lambda}$. This shorter notation is used in other cases as well, e.g. ( $X \backslash$ Int $Y, Y \backslash$ Int $Y)_{\lambda}$ stands for $\left(X_{\lambda} \backslash\right.$ Int $Y_{\lambda}, Y_{\lambda} \backslash$ Int $\left.Y_{\lambda}\right)$. In the case of triples, homotopy requirements in the definition of a map of systems are replaced by homotopy of triples. Clearly, every map of systems of triples $\mathbf{h}:(\mathbf{X}, \mathbf{Y}, \mathbf{A}) \rightarrow\left(\mathbf{X}^{\prime}, \mathbf{Y}^{\prime}, \mathbf{A}^{\prime}\right)$ determines various restrictions, e.g., $\mathbf{h} \mid(\mathbf{X}, \mathbf{Y})$ : $(\mathbf{X}, \mathbf{Y}) \rightarrow\left(\mathbf{X}^{\prime}, \mathbf{Y}^{\prime}\right), \mathbf{h} \mid \mathbf{A}: \mathbf{A} \rightarrow \mathbf{A}^{\prime}$. Whenever we speak about shape maps $\mathbf{g :} \boldsymbol{A}$ $\rightarrow A^{\prime}$ between spaces (pairs or triples of spaces), we mean by $\mathrm{g}$ a homotopy class of maps of systems $\mathbf{g}: \mathbf{A} \rightarrow \mathbf{A}^{\prime}$, where $\mathbf{A}$ and $\mathbf{A}^{\prime}$ are ANR-systems associated with $A$ and $A^{\prime}$ respectively (see [6]). In particular, $g=g^{\prime}: A \rightarrow A^{\prime}$ means that $\mathbf{g} \simeq \mathbf{g}^{\prime}: \mathbf{A} \rightarrow \mathbf{A}^{\prime}$. As usual, $\operatorname{sh} \mathbf{A}(\operatorname{sh} A)$ denotes the shape of $\mathbf{A}(A)$ and $\operatorname{sh} \mathbf{A}$ $\leq \operatorname{sh} \mathbf{A}^{\prime}\left(\operatorname{sh} A \leq \operatorname{sh} A^{\prime}\right)$ means that $\mathbf{A}^{\prime}\left(A^{\prime}\right)$ shape dominates $\mathbf{A}(A)$.

2. ANR-systems of triples with trivial middle term. The main technical result in this paper is the following theorem:

Theorem 1. Let $(\mathbf{X}, \mathbf{Y}, \mathbf{A}),\left(\mathbf{X}^{\prime}, \mathbf{Y}^{\prime}, \mathbf{A}^{\prime}\right)$ be ANR-systems of triples such that $A_{\lambda} \subset$ Int $Y_{\lambda}$ for every $\lambda \in \Lambda$ and sh $Y^{\prime}=0$. Let $\mathbf{f}:(X, Y) \rightarrow\left(X^{\prime}, Y^{\prime}\right)$ and $\mathbf{g : ~} \mathbf{A}$ $\rightarrow \mathbf{A}^{\prime}$ be maps of systems. Then there exists a map of systems of triples $\mathbf{h}:(\mathbf{X}, \mathbf{Y}, \mathbf{A})$ $\rightarrow\left(\mathbf{X}^{\prime}, \mathbf{Y}^{\prime}, \mathbf{A}^{\prime}\right)$ such that the restrictions $\mathbf{h} \mid(\mathbf{X}, \mathbf{Y}) \simeq \mathbf{f}$ and $\mathbf{h} \mid \mathbf{A} \simeq \mathbf{g}$. The map of systems $\mathbf{h}$ is unique up to homotopy.

In the proof we use repeatedly the following simple lemma:

Lemma. Let $(M, L, K),\left(M^{\prime}, L^{\prime}, K^{\prime}\right)$ be triples of metric compacta such that $K \subset$ Int $L$ and $L^{\prime}$ is an $A R$ for metric spaces. Let $\varphi_{0}, \varphi_{1}:(M, L, K) \rightarrow\left(M^{\prime}, L^{\prime}, K^{\prime}\right)$ be maps such that $\varphi_{0}\left|K \simeq \varphi_{1}\right| K$ in $K^{\prime}$,

$\varphi_{0} \mid(M \backslash$ Int $L, L \backslash$ Int $L) \simeq \varphi_{1} \mid(M \backslash$ Int $L, L \backslash$ Int $L)$ in $\left(M^{\prime}, L^{\prime}\right)$.

Then $\varphi_{0} \simeq \varphi_{1}$ in $\left(M^{\prime}, L^{\prime}, K^{\prime}\right)$.

Proof. By assumption, there exist homotopies $\Phi^{\prime}: K \times I \rightarrow K^{\prime}, \Phi^{\prime \prime}:(M \backslash$ Int $L$, $L \backslash$ Int $L) \times I \rightarrow\left(M^{\prime}, L^{\prime}\right)$ connecting

$$
\varphi_{0}\left|K, \varphi_{1}\right| K \text { and } \varphi_{0} \mid M \backslash \text { int } L, \varphi_{1} \mid M \backslash \text { Int } L
$$

respectively. Since $K \subset$ Int $L, \Phi^{\prime}, \Phi^{\prime \prime} \mid(L \backslash$ Int $L) \times I, \varphi_{0} \mid L$ and $\varphi_{1} \mid L$ define a map of a closed subset of $L \times I$ into $L^{\prime}$, which can be extended to a map $\Phi_{L}:(L, K) \times I \rightarrow\left(L^{\prime}, K^{\prime}\right)$, because $L^{\prime}$ is an AR. Since $\Phi_{L} \mid(L \backslash$ Int $L) \times I=$ $\Phi^{\prime \prime} \mid(L \backslash$ Int $L) \times I$, the maps $\Phi_{L}$ and $\Phi^{\prime \prime}$ define a homotopy $\Phi:(M, L, K) \times I$ $\rightarrow\left(M^{\prime}, L^{\prime}, K^{\prime}\right)$ connecting $\varphi_{0}$ and $\varphi_{1}$.

Proof of Theorem 1. Let $\left(\mathbf{X}^{\prime}, \mathbf{Y}^{\prime}, \mathbf{A}^{\prime}\right)=\left\{\left(X^{\prime}, Y^{\prime}, A^{\prime}\right)_{\mu}, q_{\mu \mu^{\prime}}, M\right\}$. By Lemma 5 of [9], there exists an increasing function $h: M \rightarrow \Lambda$ such that $h(\mu) \geq f(\mu), g(\mu)$. The maps $f_{\mu} p_{f(\mu) h(\mu)}$ and $g_{\mu} p_{g(\mu) h(\mu)}$, clearly, form maps of systems homotopic with $f$ and $g$ respectively. Thus, there is no loss of generality in assuming that $f=g$. 
For every $\mu \in M$ we now consider the mapping cone $C_{\mu}^{\prime}$ of the inclusion $Y_{\mu}^{\prime} \rightarrow X_{\mu}^{\prime}$ so that $C_{\mu}^{\prime}=X_{\mu}^{\prime} \cup c Y_{\mu}^{\prime}$, where $c Y_{\mu}^{\prime}$ is the cone over $Y_{\mu}^{\prime}$. Note that $c Y_{\mu}^{\prime}$ is a contractible ANR and thus an AR.

For every $\mu$ there exists a map $\chi_{\mu}:(X, Y, A)_{f(\mu)} \rightarrow\left(C^{\prime}, c Y^{\prime}, A^{\prime}\right)_{\mu}$ such that

$$
\begin{aligned}
\chi_{\mu} \mid A_{f(\mu)} & =g_{\mu}, \\
\chi_{\mu} \mid(X \backslash \operatorname{Int} Y)_{f(\mu)} & =f_{\mu} \mid(X \backslash \operatorname{Int} Y)_{f(\mu)} .
\end{aligned}
$$

Indeed, $A_{f(\mu)}$ and $(Y \backslash \text { Int } Y)_{f(\mu)}$ are disjoint closed subsets of $Y_{f(\mu)}$ and $g_{\beta}$ and $f_{\mu} \mid(Y \backslash \text { Int } Y)_{f(\mu)}$ can be extended to a map $(Y, A)_{f(\mu)} \rightarrow\left(c Y^{\prime}, A^{\prime}\right)_{\mu}$ because $c Y_{\mu}^{\prime}$ is an AR. Moreover, this map coincides with $f_{\mu}$ on $(Y \backslash \text { Int } Y)_{f(\mu)}$ and thus can be extended by $f_{\mu} \mid(X \backslash \text { Int } Y)_{f(\mu)}$ to a map $\chi_{\mu}$ having all the desired properties. For $(M, L, K)=\left(X_{f(\mu)}, Y_{f(\mu)}, \varnothing\right),\left(M^{\prime}, L^{\prime}, K^{\prime}\right)=\left(C_{\mu}^{\prime}, c Y_{\mu}^{\prime}, \varnothing\right), \varphi_{0}=\chi_{\mu}, \varphi_{1}=f_{\mu}$, the lemma yields

$$
\chi_{\mu} \simeq f_{\mu}:(X, Y)_{f(\mu)} \rightarrow\left(C_{\mu}^{\prime}, c Y_{\mu}^{\prime}\right) .
$$

We now note that for every $\mu \in M$ there is a $\psi(\mu) \geq \mu$ such that

$$
q_{\mu \psi(\mu)} \mid Y_{\psi(\mu)}^{\prime} \simeq 0
$$

This is an immediate consequence of the assumption sh $\mathbf{Y}^{\prime}=0$ (see, e.g., Theorem 5 of [6]). By Lemma 5 of [9], one can assume that $\psi: M \rightarrow \Lambda$ is an increasing function. It follows from (4) that the map $q_{\mu \psi(\mu)}$ admits an extension $q_{\mu \psi(\mu)}^{*}$ to $C_{\psi_{(\mu)}}^{\prime}$, which is a map of triples $q_{\mu \psi(\mu)}^{*}:\left(C^{\prime}, c Y^{\prime}, A^{\prime}\right)_{\psi_{(\mu)}} \rightarrow\left(X^{\prime}, Y^{\prime}, A^{\prime}\right)_{\mu}$.

We now define $h: M \rightarrow \Lambda$ by $h=f \psi$. Clearly, $h$ is an increasing function. Moreover, for every $\mu \in M$ we put

$$
h_{\mu}=q_{\mu \psi(\mu)}^{*} \chi_{\psi(\mu)}:(X, Y, A)_{h(\mu)} \rightarrow\left(X^{\prime}, Y^{\prime}, A^{\prime}\right)_{\mu} .
$$

We shall next show that for $\mu^{\prime \prime} \geq \psi(\mu)$ the following homotopy of triples holds:

$$
h_{\mu} p_{h(\mu) h\left(\mu^{\prime \prime}\right)} \simeq q_{\mu^{\prime \prime}} h_{\mu^{\prime \prime}}:(X, Y, A)_{h\left(\mu^{\prime \prime}\right)} \rightarrow\left(X^{\prime}, Y^{\prime}, A^{\prime}\right)_{\mu} .
$$

First note that

$$
\begin{aligned}
h_{\mu} p_{h(\mu) h\left(\mu^{\prime \prime}\right)} & =q_{\mu \psi(\mu)}^{*} \chi_{\psi(\mu)} p_{h(\mu) h\left(\mu^{\prime \prime}\right)}, \\
q_{\mu \mu^{\prime \prime}} h_{\mu^{\prime \prime}} & =q_{\mu \psi(\mu)} q_{\psi(\mu) \mu^{\prime \prime}} h_{\mu^{\prime \prime}}=q_{\mu \psi(\mu)}^{*} q_{\psi(\mu) \mu^{\prime \prime}} h_{\mu^{\prime \prime}},
\end{aligned}
$$

because $q_{\psi(\mu) \mu^{\mu}} h_{\mu^{\prime \prime}}\left(X_{h\left(\mu^{\mu}\right)}\right) \subset X_{\left.\psi_{\mu}\right)}^{\prime}$ and $q_{\mu \psi(\mu)}^{*} \mid X_{\psi(\mu)}^{\prime}=q_{\mu \psi(\mu)}$.

We see that the proof of (6) reduces to establishing the homotopy

$$
\chi_{\psi(\mu)} p_{h(\mu) h\left(\mu^{\prime \prime}\right)} \simeq q_{\psi(\mu) \mu^{\prime \prime}} h_{\mu^{\prime \prime}}:(X, Y, A)_{h\left(\mu^{\prime \prime}\right)} \rightarrow\left(C^{\prime}, c Y^{\prime}, A^{\prime}\right)_{\psi(\mu)}
$$


Now (1) and (5) imply

$$
\begin{aligned}
\chi_{\psi(\mu)} p_{h(\mu) h\left(\mu^{\prime \prime}\right)} \mid A_{h\left(\mu^{\prime \prime}\right)} & =g_{\psi(\mu)} p_{h(\mu) h\left(\mu^{\prime \prime}\right)} \mid A_{h\left(\mu^{\prime \prime}\right)}, \\
q_{\psi(\mu) \mu^{\prime \prime}} h_{\mu^{\prime \prime}} \mid A_{h\left(\mu^{\prime \prime}\right)} & =q_{\psi(\mu) \mu^{*}} q_{\mu^{\prime \prime} \psi\left(\mu^{\prime \prime}\right)} g_{\psi\left(\mu^{\prime \prime}\right)}=q_{\psi(\mu) \psi\left(\mu^{\prime \prime}\right)} g_{\psi\left(\mu^{\prime \prime}\right),}
\end{aligned}
$$

and since $\mathbf{g :} \mathbf{A} \rightarrow \mathbf{A}^{\prime}$ is a map of systems we have

$$
\chi_{\psi(\mu)} p_{h(\mu) h\left(\mu^{*}\right)}\left|A_{h\left(\mu^{*}\right)} \simeq q_{\psi(\mu) \mu^{*}} h_{\mu^{*}}\right| A_{h\left(\mu^{*}\right)} \text { in } A_{\psi(\mu)}^{\prime} \text {. }
$$

On the other hand, by (3),

$$
\begin{aligned}
& \chi_{\psi(\mu)} p_{h(\mu) h\left(\mu^{*}\right)} \mid(X \backslash \text { Int } Y, Y \backslash \text { Int } Y)_{h\left(\mu^{*}\right)} \\
& \simeq f_{\psi(\mu)} p_{h(\mu) h\left(\mu^{*}\right)} \mid(X \backslash \text { Int } Y, Y \backslash \text { Int } Y)_{h\left(\mu^{*}\right)} \text { in }\left(C^{\prime}, c Y^{\prime}\right)_{\Psi(\mu)} .
\end{aligned}
$$

Moreover, by (5) and (2),

$$
q_{\psi(\mu) \mu^{*}} h_{\mu^{*}} \mid(X \backslash \text { Int } Y)_{h\left(\mu^{*}\right)}=q_{\psi(\mu) \psi\left(\mu^{*}\right)} f_{\psi\left(\mu^{*}\right)} \mid(X \backslash \text { Int } Y)_{h\left(\mu^{*}\right)} .
$$

Since $\mathbf{f :}(\mathbf{X}, \mathbf{Y}) \rightarrow\left(\mathbf{X}^{\prime}, \mathbf{Y}^{\prime}\right)$ is a map of systems, there is a homotopy

$$
f_{\psi(\mu)} p_{h(\mu) h\left(\mu^{\prime \prime}\right)} \simeq q_{\psi(\mu) \psi\left(\mu^{\prime \prime}\right)} f_{\psi\left(\mu^{\prime \prime}\right)} \text { in }\left(X^{\prime}, Y^{\prime}\right)_{\psi(\mu)} \subset\left(C^{\prime}, c Y^{\prime}\right)_{\psi(\mu)}
$$

We apply now the lemma, by putting $(M, L, K)=(X, Y, A)_{\left(m^{*}\right)},\left(M^{\prime}, L^{\prime}, K^{\prime}\right)$ $=\left(C^{\prime}, c Y^{\prime}, A^{\prime}\right)_{\psi(\mu)}, \varphi_{0}=\chi_{\psi(\mu)} p_{h(\mu) h\left(\mu^{\prime \prime}\right)}, \varphi_{1}=q_{\psi(\mu) \mu^{*}} h_{\mu^{\prime \prime}}$, and we obtain (9). Formula (6) is thus established.

We now observe that for arbitrary $\mu \leq \mu^{\prime}$ there exists a $\mu^{\prime \prime} \geq \psi\left(\mu^{\prime}\right) \geq \psi(\mu)$. Hence, by (6),

$$
h_{\mu} p_{h(\mu) h\left(\mu^{\prime \prime}\right)} \simeq q_{\mu \mu^{*}} h_{\mu^{\prime \prime}}=q_{\mu \mu^{\prime}} q_{\mu^{\prime} \mu^{*}} h_{\mu^{*}} \simeq q_{\mu^{\prime}} h_{\mu^{\prime}} p_{h\left(\mu^{\prime}\right) h\left(\mu^{\prime}\right)}
$$

Although the maps $h_{\mu}$ need not form a map of systems $(\mathbf{X}, \mathbf{Y}, \mathbf{A}) \rightarrow\left(\mathbf{X}^{\prime}, \mathbf{Y}^{\prime}, \mathbf{A}^{\prime}\right)$ in the sense of [9], it follows from (16) and Lemma 7 of [9] that for a suitable increasing function $h^{*}: M \rightarrow \Lambda, h^{*} \geq h$, the maps $h_{\mu} p_{h(\mu) h^{*}(\mu)}$ form a map of systems h: $(\mathbf{X}, \mathbf{Y}, \mathbf{A}) \rightarrow\left(\mathbf{X}^{\prime}, \mathbf{Y}^{\prime}, \mathbf{A}^{\prime}\right)$.

It follows from (5) and (3) that

$$
\begin{aligned}
h_{\mu} p_{h(\mu) h^{*}(\mu)} \mid(X, Y)_{h^{*}(\mu)} & \simeq q_{\mu \psi(\mu)} f_{\psi(\mu)} p_{h(\mu) h^{*}(\mu)} \mid(X, Y)_{h^{*}(\mu)} \\
& \simeq f_{\mu} p_{f(\mu) h^{*}(\mu)} \mid(X, Y)_{h^{*}(\mu)} \text { in }\left(X^{\prime}, Y^{\prime}\right)_{\mu},
\end{aligned}
$$

which shows that $\mathbf{h} \mid(\mathbf{X}, \mathbf{Y}) \simeq \mathbf{f}$.

Finally, by (5) and (1),

$$
\begin{aligned}
h_{\mu} p_{h(\mu) h^{*}(\mu)} \mid A_{h^{*}(\mu)} & =q_{\mu \psi(\mu)} g_{\psi(\mu)} p_{h(\mu) h^{*}(\mu)} \mid A_{h^{*}(\mu)} \\
& \simeq g_{\mu} p_{f(\mu) h^{*}(\mu)} \mid A_{h^{*}(\mu)} \quad \text { in } A_{\mu}^{\prime} .
\end{aligned}
$$


which shows that $\mathbf{h} \mid \mathbf{A} \simeq \mathbf{g}$. This completes the proof of the existence of $\mathbf{h}$.

We now prove the uniqueness of $h$. Assume that $h, k:(X, Y, A) \rightarrow\left(X^{\prime}, Y^{\prime}, A^{\prime}\right)$ are two maps of systems satisfying the assertion of the theorem. Since $\mathbf{h} \mid \mathbf{A} \simeq \mathbf{g}$ $\simeq \mathbf{k} \mid \mathbf{A}: \mathbf{A} \rightarrow \mathbf{A}^{\prime}$, for every $\mu \in M$ there is a $\lambda \geq h \psi(\mu), k \psi(\mu)$ such that

$$
h_{\psi(\mu)} p_{h \psi(\mu) \lambda}\left|A_{\lambda} \simeq k_{\psi(\mu)} p_{k \psi(\mu) \lambda}\right| A_{\lambda} \text { in } A_{\psi(\mu)}^{\prime} .
$$

Furthermore, $\mathbf{h}|(\mathbf{X}, \mathbf{Y}) \simeq \mathbf{f} \simeq \mathbf{k}|(\mathbf{X}, \mathbf{Y})$, which means that for every $\mu \in M$ there is a $\lambda \geq h \psi(\mu), k \psi(\mu)$ such that

(20) $h_{\psi(\mu)} p_{h_{\psi(\mu) \lambda}}\left|(X, Y)_{\lambda} \simeq k_{\psi(\mu)} p_{k \psi(\mu) \lambda}\right|(X, Y)_{\lambda}$ in $\left(X^{\prime}, Y^{\prime}\right)_{\left.\psi_{\mu}\right)} \subset\left(C^{\prime}, c Y^{\prime}\right)_{\left.\psi_{\mu}\right)}$.

There is no loss of generality in taking the same $\lambda$ in (19) and (20). Applying the lemma with $(M, L, K)=(X, Y, A)_{\lambda}, \quad\left(M^{\prime}, L^{\prime}, K^{\prime}\right)=\left(C^{\prime}, c Y^{\prime}, A^{\prime}\right)_{\left.\psi_{\mu}\right)}, \quad \varphi_{0}$ $=h_{\psi(\mu)} p_{h_{\psi(\mu) \lambda},}, \varphi_{1}=k_{\psi(\mu)} p_{k \psi(\mu) \lambda}$, one obtains

$$
h_{\psi(\mu)} p_{h \psi(\mu) \lambda} \simeq k_{\psi(\mu)} p_{k \psi(\mu) \lambda} \text { in }\left(C^{\prime}, c Y^{\prime}, A^{\prime}\right)_{\left.\psi_{\mu}\right)},
$$

and composition with $q_{\mu \psi(\mu)}^{*}$ yields

$$
q_{\mu \psi(\mu)} h_{\psi(\mu)} p_{h \psi(\mu) \lambda} \simeq q_{\mu \psi(\mu)} k_{\psi(\mu)} p_{k \psi(\mu) \lambda} \text { in }\left(X^{\prime}, Y^{\prime}, A^{\prime}\right)_{\psi(\mu)} .
$$

On the other hand, since $h$ and $k$ are maps of systems, we have

$$
\begin{aligned}
& h_{\mu} p_{h(\mu) \lambda} \simeq q_{\mu \psi(\mu)} h_{\psi(\mu)} p_{h \psi(\mu) \lambda}, \\
& k_{\mu} p_{k(\mu) \lambda} \simeq q_{\mu \psi(\mu)} k_{\psi(\mu)} p_{k \psi(\mu) \lambda},
\end{aligned}
$$

so that (22) implies

$$
h_{\mu} p_{h(\mu) \lambda} \simeq k_{\mu} p_{k(\mu) \lambda} \text {. }
$$

This proves that $\mathbf{h} \simeq \mathbf{k}$ and completes the proof of Theorem 1 .

3. Triples of compacta with trivial middle term. We now state and prove the corresponding theorem for spaces.

Theorem 2. Let $(X, Y, A),\left(X^{\prime}, Y^{\prime}, A^{\prime}\right)$ be triples of compact Hausdorff spaces such that sh $Y^{\prime}=0$. Let $\mathrm{f}:(X, Y) \rightarrow\left(X^{\prime}, Y^{\prime}\right)$ and $\mathrm{g}: A \rightarrow A^{\prime}$ be shape maps. Then there exists a unique shape map h: $(X, Y, A) \rightarrow\left(X^{\prime}, Y^{\prime}, A^{\prime}\right)$ such that $\mathbf{h} \mid(X, Y)=\mathbf{f}$ and h $\mid \boldsymbol{A}=\mathbf{g}$.

Proof. We first observe that there exists an ANR-system (X, Y, A) associated with $(X, Y, A)$ and such that $A_{\lambda} \subset \operatorname{Int} Y_{\lambda}$ for every $\lambda \in \Lambda$. Such a system is obtained by suitably modifying the construction in the proof of Theorem 7 of [9]. With $\left(X^{\prime}, Y^{\prime}, A^{\prime}\right)$ we associate an arbitrary ANR-system expansion $\left(\mathbf{X}^{\prime}, \mathbf{Y}^{\prime}, \mathbf{A}^{\prime}\right)$. 
By assumption, sh $\mathbf{Y}^{\prime}=0$ and we have maps of systems $f:(X, Y) \rightarrow\left(X^{\prime}, Y^{\prime}\right)$, g: $A \rightarrow A^{\prime}$. Then Theorem 1 yields a map of systems $h:(X, Y, A) \rightarrow\left(X^{\prime}, Y^{\prime}, A^{\prime}\right)$, which restricted to $(X, Y)$ and $A$ is homotopic to $\mathbf{f}$ and $\mathbf{g}$ respectively. Consequently, we obtain a shape map h: $(X, Y, A) \rightarrow\left(X^{\prime}, Y^{\prime}, A^{\prime}\right)$ satisfying the assertions of Theorem 2.

Remark 2. In the case that $\left(X^{\prime}, Y^{\prime}, A^{\prime}\right)$ is metric, a considerably simpler proof of Theorem 2 can be given provided one is willing to use the main result of [7]. In that case, one can obtain, namely, an ANR-expansion $\left(\mathbf{X}^{\prime}, \mathbf{Y}^{\prime}, \mathbf{A}^{\prime}\right)$ where all $Y_{\mu}^{\prime}$ are Hilbert cubes and thus AR's. The proof of [7] uses, however, techniques of PL-topology.

Theorem 3. Let $(X, Y, A),\left(X^{\prime}, Y^{\prime}, A^{\prime}\right)$ be triples of compact Hausdorff spaces with $\operatorname{sh} Y=\operatorname{sh} Y^{\prime}=0$. Then the following assertions hold:

(i) $\operatorname{sh}(X, Y) \leq \operatorname{sh}\left(X^{\prime}, Y^{\prime}\right)$ and $\operatorname{sh} A \leq \operatorname{sh} A^{\prime}$ imply $\operatorname{sh}(X, Y, A) \leq \operatorname{sh}\left(X^{\prime}, Y^{\prime}, A^{\prime}\right)$.

(ii) $\operatorname{sh}(X, Y)=\operatorname{sh}\left(X^{\prime}, Y^{\prime}\right)$ and $\operatorname{sh} A=\operatorname{sh} A^{\prime}$ imply $\operatorname{sh}(X, Y, A)=\operatorname{sh}\left(X^{\prime}, Y^{\prime}, A^{\prime}\right)$.

Proof. By assumption there exist shape maps $f:(X, Y) \rightarrow\left(X^{\prime}, Y^{\prime}\right), f^{\prime}:\left(X^{\prime}, Y^{\prime}\right)$ $\rightarrow(X, Y), \mathrm{g}: A \rightarrow A^{\prime}, \mathbf{g}^{\prime}: A^{\prime} \rightarrow A$ such that

$$
\begin{array}{r}
\mathbf{f}^{\prime} \mathbf{f}=\mathbf{1}, \\
g^{\prime} \mathbf{g}=\mathbf{1}
\end{array}
$$

By Theorem 2, there exist shape maps $\mathrm{h}:(X, Y, A) \rightarrow\left(X^{\prime}, Y^{\prime}, A^{\prime}\right), \mathrm{h}^{\prime}:\left(X^{\prime}, Y^{\prime}, A^{\prime}\right)$ $\rightarrow(X, Y, A)$ such that

$$
\begin{array}{rr}
\mathbf{h} \mid(X, Y=\mathbf{f}, & \mathbf{h} \mid A=\mathbf{g}, \\
\mathbf{h}^{\prime} \mid\left(X^{\prime}, Y^{\prime}\right)=\mathbf{f}^{\prime} & \mathbf{h}^{\prime} \mid A^{\prime}=\mathbf{g}^{\prime} .
\end{array}
$$

Consequently $\mathbf{h}^{\prime} \mathbf{h}:(X, Y, A) \rightarrow(X, Y, A)$ satisfies

$$
\begin{array}{r}
\mathbf{h}^{\prime} \mathbf{h} \mid(X, Y)=\mathbf{f}^{\prime} \mathbf{f}=\mathbf{1}, \\
\mathbf{h}^{\prime} \mathbf{h} \mid A=\mathbf{g}^{\prime} \mathbf{g}=\mathbf{1} .
\end{array}
$$

Since 1: $(X, Y, A) \rightarrow(X, Y, A)$ also has these properties, it follows from the uniqueness (Theorem 2), that

$$
\mathbf{h}^{\prime} \mathbf{h}=\mathbf{x},
$$

which establishes $\operatorname{sh}(X, Y, A) \leq \operatorname{sh}\left(X^{\prime}, Y^{\prime}, A^{\prime}\right)$.

The proof of (ii) is analogous.

It was proved in [5] that $\operatorname{sh}(Y, A)=\operatorname{sh}\left(Y^{\prime}, A^{\prime}\right)$ implies $\operatorname{sh}(Y / A)=\operatorname{sh}\left(Y^{\prime} / A^{\prime}\right)$.

The same proof also establishes the following more general result: 
Theorem 4. Let $\mathrm{h}:(X, Y, A) \rightarrow\left(X^{\prime}, Y^{\prime}, A^{\prime}\right)$ be a shape map and let $p:(X, Y, A)$ $\rightarrow(X / A, Y / A, A / A), p^{\prime}:\left(X^{\prime}, Y^{\prime}, A^{\prime}\right) \rightarrow\left(X^{\prime} / A^{\prime}, Y^{\prime} / A^{\prime}, A^{\prime} / A^{\prime}\right)$ be quotient maps. Then there is a unique shape map $\mathrm{h}^{*}:(X / A, Y / A, A / A) \rightarrow\left(X^{\prime} / A^{\prime}, Y^{\prime} / A^{\prime}, A^{\prime} / A^{\prime}\right)$ such that $\mathbf{p}^{\prime} \mathbf{h}=\mathbf{h}^{*} \mathbf{p}$. Moreover, if $\mathbf{h}^{\prime}:\left(X^{\prime}, Y^{\prime}, A^{\prime}\right) \rightarrow\left(X^{\prime \prime}, Y^{\prime \prime}, A^{\prime \prime}\right)$ is another shape map, then $\left(\mathbf{h}^{\prime} \mathbf{h}\right)^{*}=\mathbf{h}^{\prime *} \mathbf{h}^{*}, \quad \mathbf{1}^{*}=1$. Consequently, $\operatorname{sh}(X, Y, A) \leq \operatorname{sh}\left(X^{\prime}, Y^{\prime}, A^{\prime}\right)$ implies $\operatorname{sh}(X / A, Y / A, A / A) \leq \operatorname{sh}\left(X^{\prime} / A^{\prime}, Y^{\prime} / A^{\prime}, A^{\prime} / A^{\prime}\right)$ and $\operatorname{sh}(X, Y, A)=\operatorname{sh}\left(X^{\prime}, Y^{\prime}, A^{\prime}\right)$ implies $\operatorname{sh}(X / A, Y / A, A / A)=\operatorname{sh}\left(X^{\prime} / A^{\prime}, Y^{\prime} / A^{\prime}, A^{\prime} / A^{\prime}\right)$.

4. Corollaries. We now derive from Theorems 3 and 4 a number of corollaries. Some of these results, when restricted to the metric case, become known theorems proved by Borsuk or Chapman. For simplicity we omit the results on shape domination.

Corollary 1. Let $(X, Y)$ be a compact Hausdorff pair and $A, A^{\prime}$ closed subsets of $Y$. If $\operatorname{sh} A=\operatorname{sh} A^{\prime}$ and $\operatorname{sh} Y=0$, then $\operatorname{sh}(X, Y, A)=\operatorname{sh}\left(X, Y, A^{\prime}\right)$ and thus $\operatorname{sh}(X, A)=\operatorname{sh}\left(X, A^{\prime}\right)$ and $\operatorname{sh}(X / A)=\operatorname{sh}\left(X / A^{\prime}\right)$.

This generalizes Chapman's Theorem 1 of [4] and gives a positive answer to problem (5.4) of [2] (for shape in the sense of ANR-systems).

Corollary 2. Let $(X, A)$ be a compact Hausdorff pair such that $\operatorname{sh} A=0$ and let $a_{0}$ be any point of $A$. Then $\operatorname{sh}(X, A)=\operatorname{sh}\left(X, a_{0}\right)$ and thus $\operatorname{sh}(X / A, A / A)=\operatorname{sh}\left(X, a_{0}\right)$ and $\operatorname{sh}(X / A)=\operatorname{sh} X$.

Proof. Putting $Y=A, A^{\prime}=\left\{a_{0}\right\}$ and applying Corollary 1 , one obtains $\operatorname{sh}(X, A)=\operatorname{sh}\left(X, a_{0}\right)$. Now Theorem 4 yields $\operatorname{sh}(X / A, A / A)=\operatorname{sh}\left(X, a_{0}\right)$ and therefore also $\operatorname{sh}(X / A)=\operatorname{sh} X$.

Corollary 3. Let $(X, Y, A),\left(X^{\prime}, Y^{\prime}, A^{\prime}\right)$ be triples of compact Hausdorff spaces. If $\operatorname{sh} Y=\operatorname{sh} Y^{\prime}=0, \operatorname{sh}(X / Y, Y / Y)=\operatorname{sh}\left(X^{\prime} / Y^{\prime}, Y^{\prime} / Y^{\prime}\right)$ and $\operatorname{sh} A=\operatorname{sh} A^{\prime}$, then $\operatorname{sh}(X, Y, A)=\operatorname{sh}\left(X^{\prime}, Y^{\prime}, A^{\prime}\right)$ and thus $\operatorname{sh}(X / A)=\operatorname{sh}\left(X^{\prime} / A^{\prime}\right)$.

Proof. By Corollary 2, $\operatorname{sh}(X / Y, Y / Y)=\operatorname{sh}(X, Y), \operatorname{sh}\left(X^{\prime} / Y^{\prime}, Y^{\prime} / Y^{\prime}\right)=$ $\operatorname{sh}\left(X^{\prime}, Y^{\prime}\right)$, so that $\operatorname{sh}(X, Y)=\operatorname{sh}\left(X^{\prime}, Y^{\prime}\right)$. Now Theorem 3 implies $\operatorname{sh}(X, Y, A)$ $=\operatorname{sh}\left(X^{\prime}, Y^{\prime}, A^{\prime}\right)$. This generalizes Chapman's Theorem 2 of [4].

Corollary 4. Let $X^{\prime}, X^{\prime \prime}$ be compact Hausdorff spaces and let $X=X^{\prime} \cup X^{\prime \prime}$, $X^{0}=X^{\prime} \cap X^{\prime \prime}$. Then the following assertions hold:

(i) $\operatorname{sh} X^{\prime}=\operatorname{sh} X^{\prime \prime}=\operatorname{sh} X^{0}=0 \Rightarrow \operatorname{sh} X=0$.

(ii) $\operatorname{sh} X^{0}=\operatorname{sh} X=0 \Rightarrow \operatorname{sh} X^{\prime}=\operatorname{sh} X^{\prime \prime}=0$.

Proof. Let $Y=X / X^{0}, Y^{\prime}=X^{\prime} / X^{0}, Y^{\prime \prime}=X^{\prime \prime} / X^{0}$. Then $Y=Y^{\prime} \vee Y^{\prime \prime}$ is the wedge of $Y^{\prime}$ and $Y^{\prime \prime}$. By the assumptions in (i) and (ii) $\operatorname{sh} X^{0}=0$, so that Corollary 2 implies

$$
\operatorname{sh} Y^{\prime}=\operatorname{sh} X^{\prime}, \quad \operatorname{sh} Y^{\prime \prime}=\operatorname{sh} X^{\prime \prime}, \quad \operatorname{sh} Y=\operatorname{sh} X .
$$


Furthermore, $Y^{\prime}$ and $Y^{\prime \prime}$ are retracts of $Y$ and so

$$
\operatorname{sh} Y^{\prime} \leq \operatorname{sh} Y, \quad \operatorname{sh} Y^{\prime \prime} \leq \operatorname{sh} Y .
$$

Case (i). Since $Y^{\prime}=Y / Y^{\prime \prime}$ and, by (1), sh $Y^{\prime \prime}=\operatorname{sh} X^{\prime \prime}=0$, Corollary 2 implies sh $Y=\operatorname{sh} Y^{\prime}$. Hence, by (1), sh $X=\operatorname{sh} X=\operatorname{sh} Y^{\prime}=\operatorname{sh} X^{\prime}=0$.

Case (ii). By (1) and (2), $\operatorname{sh} X^{\prime}=\operatorname{sh} Y^{\prime} \leq \operatorname{sh} Y=\operatorname{sh} X=0$, so that $\operatorname{sh} X^{\prime}$ $=0$. Similarly, sh $X^{\prime \prime}=0$.

(i) is Borsuk's Theorem 14.1 of [1], and (ii) is Chapman's Theorem 1 of [3], both generalized to nonmetric compacta.

Corollary 5. Let $(X, A)$ be a compact pair, $Y$ a compact space, and $f: A \rightarrow Y$ a map. If $\operatorname{sh} X=\operatorname{sh} A=\operatorname{sh} Y=0$, then the adjunction space $Z=X \cup_{f} Y$ is also of trivial shape.

Proof. Since $Y \subset Z$ and sh $Y=0$, Corollary 2 implies $\operatorname{sh}(Z / Y)=\operatorname{sh} Z$. However, $Z / Y$ is homeomorphic with $X / A$ and since $\operatorname{sh} A=0$, we obtain $\operatorname{sh}(Z / Y)=\operatorname{sh}(X / A)=\operatorname{sh} X=0$. Hence, $\operatorname{sh} Z=0$.

The metric version of Corollary 5 is Theorem 2 of [3].

\section{REFERENCES}

1. K. Borsuk, Fundamental retracts and extensions of fundamental sequences, Fund. Math. 64 (1969), 55-85. MR 39 \# 4841.

2.—, Remark on a theorem of S. Mardešić, Bull. Acad. Polon. Sci. Sér. Sci. Math. Astronom. Phys. 19 (1971), 475-483.

3. T. A. Chapman, Some results on shapes and fundamental absolute retracts, Bull. Acad. Polon. Sci. Sér. Sci. Math. Astronom. Phys. 20 (1972), 37-40.

4. - Shapes of some decomposition spaces, Bull. Acad. Polon. Sci. Ser. Sci. Math. Astronom. Phys. 20 (1972), 653-656.

5. S. Mardešic, On the shape of the quotient space $S^{n} / A$, Bull. Acad. Polon. Sci. Sér. Sci. Math. Astronom. Phys. 19 (1971), 623-629.

6.—, Retracts in shape theory, Glasnik Mat. 6 (26) (1971), 153-163. MR 45 \# 5974.

7. Decreasing sequences of cubes and compacta of trivial shape, General Topology Appl. 2 (1972), 17-23.

8.—, On Borsuk's shape theory for compact pairs, Bull. Acad. Polon. Sci. Sér. Sci. Math. Astronom. Phys. 21 (1973), 13-18.

9. S. Mardesić and J. Segal, Shapes of compacta and ANR-systems, Fund. Math. 72 (1971), 41-59. MR 45 \# 7686.

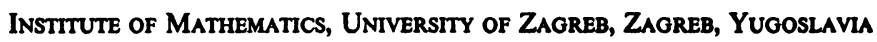

